\title{
Effects of Procainmide and Lidocaine on Electrically Inducible Ventricular Tachycardia Studied with Programmed Ventricular Stimulation in Post Myocardial Infarction
}

\author{
Yoshito Iesaka, M.D., Kazutaka Aonuma, M.D., Junichi Nitta, M.D. \\ Takeshi Tokunaga, M.D., Hideomi Fujiwara, M.D. \\ and Masayasu Hiraoka, M.D*
}

\begin{abstract}
The effects of procainamide and lidocaine, representative of class IA and IB antiarrhythmic agents, on electrically inducible ventricular tachycardia (VT) were studied using programmed ventricular stimulation in 47 post myocardial infarction patients at an average of 1.5 months after the onset. The mean doses of administered procainamide and lidocaine were $1050 \mathrm{mg}$ and $161 \mathrm{mg}$, and their mean plasma concentrations were $7.5 \mu \mathrm{g} / \mathrm{ml}$ and $3.1 \mu \mathrm{g} / \mathrm{ml}$ respectively. The induction of sustained VT was suppressed in 15 of 29 patients $(52 \%)$ by procainamide, but in none by lidocaine. The induction of nonsustained VT was suppressed in 6 of 18 patients $(33 \%)$ by procainamide, and in 1 of 8 patients $(13 \%)$ by lidocaine. The efficacy rate of procainamide was significantly higher than that of lidocaine in suppression of VT induction $(21 / 47$ vs $1 / 14 p<0.01)$. Procainamide significantly prolonged the effective refractory period of the right ventricle as well as the HV and QRS interval, however lidocaine did not affect them significantly. On the other hand, the worsening effect which changed nonsustained VT inducible in the baseline into sustained VT inducible post drug administration was demonstrated in 8 of 18 procainamide cases $(44 \%)$, and in 3 of 8 lidocaine cases $(38 \%)$. Between the procainamide effective and ineffective or worsening patients, there were no differences found in the electrophysiologic variables either in the baseline or post procainamide administration.

We concluded that procainamide was more effective than lidocaine for the prevention of potential life-threatening VT induction in post myocardial infarction patients, although its efficacy was considerably limited, and to confirm the effectiveness and exclude the worsening effects of the class IA and IB antiarrhythmic agents, drug testing using programmed ventricular stimulation appeared to be valuable.
\end{abstract}

Key words:

Antiarrhy thmic effect

Proarrhythmic effect

Electrically inducible ventricular tachycardia

Programmed ventricular stimulation

Post myocardial infarction
$\mathbf{T}$

HE risk of sudden cardiac death is high in survivors of acute myocardial infarction, and its major cause has been indicated to be lifethreatening ventricular tachyarrhythmias. ${ }^{1-4}$ Frequent and repetitive ventricular premature complexes detected by ambulatory electrocardiographic monitoring and left ventricular

Department of Cardiology, Tsuchiura Kyodo Hospital, Ibaraki; ${ }^{*}$ Department of Cardiovascular Disease, Medical Research Institute, Tokyo Medical and Dental University, Tokyo, Japan

Mailing address: Yoshito Iesaka, M.D., Department of Cardiology, Tsuchiura Kyodo Hospital, 11-7 Manabeshinmachi, Tsuchiura-shi, Ibaraki-ken 300, Japan 
dysfunction have been reported to be predictors of increased mortality.-7 However the predictive accuracy of these markers of the risk for sudden death is not necessarily high, and their interpretation is still somewhat controversial in the literature. ${ }^{4}$

Recently, programmed ventricular stimulation of the heart has been used to identify patients at high risk of sudden cardiac death or spontaneous development of ventricular tachycardia following myocardial infarction. ${ }^{8-20}$ This is based on the idea that sudden cardiac death after acute myocardial infarction is caused, in the majority of cases, by spontaneous occurrence of sustained ventricular tachycardia or fibrillation and that programmed ventricular stimulation is effective in disclosing the substrate which initiates and perpetuates those reentrant sustained ventricular tachyarrhythmias with or without clinical documentations of spontaneously occurring arrhythmias. ${ }^{8-20}$ Programmed ventricular stimulation was initially used in the diagnosis and management of recurrent sustained ventricular tachycardia. The usefulness of this technique in evaluating the efficacy of antiarrhythmic agents on electrically inducible ventricular tachycardia has also been well appreciated? ${ }^{2-23}$

In this study using programmed ventricular stimulation, we investigated the effects of the most widely used class IA and IB antiarrhythmic agents on electrically inducible ventricular tachycardias in potential high risk patients of post myocardial infarction. We aimed to evaluate the efficacy and limitations of treatment with conventional antiarrhythmic agents in preventing sudden cardiac death and life-threatening ventricular tachyarrhythmias. We also studied the utility of drug testing using programmed ventricular stimulation.

\section{METHODS}

The subjects in this study were 47 patients (42 males and 5 females) associated with ventricular tachycardia (VT) induced by programmed ventricular stimulation. Programmed ventricular stimulation was performed in 120 patients at an average of 1.5 months (1-6 months) after acute myocardial infarction in an attempt to identify high risk patients for sudden cardiac death. The mean age was 53 years, and mean left ventricular ejection fraction was $51 \pm 14 \%$ (mean \pm SD).

Informed consent was obtained following an explanation of the significance and risks of VT induction study. Then patients underwent baseline electrophysiologic study in the fasting state under light diazepam sedation. All the antiarrhythmic agents were discontinued at least 5 half-lives in the plasma before study. Following standard electrophysiologic technique, 2 quadripolar electrode cathethers were inserted percutaneously through a femoral vein and positioned at the right ventricular apex and outflow tract to stimulate the heart and record local endocardial electrograms. Another catheter was placed at the His region for recording the His-bundle electrogram or at the high right atrium for atrioventricular sequential pacing. Electrocardiographic leads I, II and V1, and 5 traces of intracardiac electrograms recorded at the right ventricular apex, outflow tract, His region or high right atrium were displayed on an oscilloscope and recorded with an ink-jet recorder (Siemens-Elema Mingograf 82) at a paper speed of 50 or $100 \mathrm{~mm} / \mathrm{sec}$. All these records were stored on magnetic tape by a data recorder. Cardiac stimulation was performed with rectangular pulses of $1 \mathrm{~ms}$ duration at a stimulus strength of twice diastolic threshold using a programmable stimulator (FUKUDA Cardiac Stimulator BC-02).

The programmed ventricular stimulation was performed with 2 basic drive cycle lengths ( 400 and $600 \mathrm{~ms}$ ) of 5 beats followed by extrastimuli. Single and then double extrastimuli were introduced from the right ventricular apex and outflow tract. If sustained VT was not induced, triple extrastimuli were introduced from both right ventricular sites. The end point of stimulation protocol was the induction of sustained VT or completion of the protocol.

After the completion of the baseline study, the drug effect was tested in the patients with VT inducible using the same stimulation protocol after the application of the drug. Procainamide was administered intravenously at a rate of $50 \mathrm{mg} / \mathrm{min}$ up to a total dose of $1-2 \mathrm{~g}$. Lidocaine was administered intravenously at a dose of $2-3 \mathrm{mg} / \mathrm{kg}$ and a rate of $30 \mathrm{mg} / \mathrm{min}$, followed by a continuous infusion at a rate of $1-3 \mathrm{mg} /$ min. In 34 cases, either procainamide or lidocaine was tested. The combined effects of procainamide and lidocaine were studied in 3 cases when lidocaine was administered immediately after procainamide testing was completed. In the remaining 13 cases lidocaine and then procainamide, which was administered $1 \mathrm{hr}$ after the completion of lidocaine testing, were tested

Japanese Circulation Journal Vol. 52, March I 988 
TABLE I PERTINENT ELECTROPHYSIOLOGIC DATA OF THE INDUCED VENTRICULAR TACHYCARDIA (VT)

\begin{tabular}{|c|c|c|c|c|c|c|c|c|}
\hline & \multirow{2}{*}{$\begin{array}{c}\text { No. of } \\
\text { cases }\end{array}$} & \multirow{2}{*}{$\begin{array}{c}V T C L \\
(m s)\end{array}$} & \multicolumn{4}{|c|}{ Initiation mode } & \multicolumn{2}{|c|}{ Termination mode } \\
\hline & & & $S_{2}$ & $S_{2} S_{3}$ & $S_{2} S_{3} S_{4}$ & burst VP & burst VP & DC shock \\
\hline Sustained VT & 29 & $\begin{array}{c}238 \pm 66 \\
(\text { mean } \pm S D)\end{array}$ & 2 & 7 & 19 & 1 & 14 & 15 \\
\hline Polymorphic & 4 & $193 \pm 15$ & 0 & 1 & 3 & 0 & 0 & 4 \\
\hline Monomorphic & 25 & $245 \pm 61$ & 2 & 6 & 16 & 1 & 14 & 11 \\
\hline Nonsustained VT & 18 & $235 \pm 27$ & 0 & 3 & 15 & 0 & \multicolumn{2}{|c|}{ spontaneous } \\
\hline Polymorphic & 9 & $218 \pm 26$ & 0 & 3 & 6 & 0 & \multicolumn{2}{|c|}{ spontaneous } \\
\hline Monomorphic & 9 & $243 \pm 21$ & 0 & 0 & 9 & 0 & \multicolumn{2}{|c|}{ spontaneous } \\
\hline
\end{tabular}

$V T=$ ventricular tachycardia $\quad V P=$ ventricular pacing; $D C=$ direct current $; C L=$ cycle length

TABLE II SUPPRESSIVE EFFECTS OF PROCAINAMIDE AND LIDOCAINE ON VT INDUCTION

\begin{tabular}{cccc}
\hline \hline Type of VT & Procainamide & Lidocaine & \\
\hline Sustained VT & $15 / 29(52 \%)$ & $0 / 6(0 \%)$ & $p<0.024$ \\
Nonsustained VT & $6 / 18(33 \%)$ & $1 / 8(13 \%)$ & $p<0.027$ \\
Total & $21 / 47(45 \%)$ & $1 / 14(7 \%)$ & $p<0.011$ \\
\hline
\end{tabular}

$V T=$ ventricular tachycardia

respectively. Plasma concentration of the drug was measured immediately after the testing was completed.

For statistical analysis, paired and unpaired $t$ test and Fisher's exact test were used. A p value $<0.05$ was considered significant. Values are expressed as mean \pm standard deviation.

Sustained VT was defined as VT lasting longer than 30 seconds or requiring termination before that period because of hemodynamic compromise. Nonsustained VT was defined as 10 or more consecutive ventricular beats terminating spontaneously in less than 30 seconds. Induced VT was classified into monomorphic VT with constant QRS configuration and axis, and polymorphic VT with changing QRS configuration and ax is in successive beats.

The drug effects were judged as effective when nonsustained VT changed into no VT inducible or sustained VT changed into no VT or nonsustained VT inducible after the drug administration. A worsening effect of the drugs was defined as nonsustained VT changed into sustained VT inducible.

\section{RESULTS}

The pertinent data on induced VT at baseline programmed ventricular stimulation are shown in Table I. Fourty seven VTs consisted of 25 sustained monomorphic, 4 sustained polymorphic, 9 nonsustained monomorphic and 9 nonsustained polymorphic VT. The mean cycle lengths of induced VT were $238 \pm 66 \mathrm{~ms}$ in sustained VT and $235 \pm 27 \mathrm{~ms}$ in nonsustained VT. Mostly triple ex trastimuli were needed to induce VT.

The mean doses of procainamide and lidocaine were $1050 \mathrm{mg}$ and $161 \mathrm{mg}$, respectively. The mean plasma concentrations of procainamide and lidocaine were $7.5 \mu \mathrm{g} / \mathrm{ml}$ and $3.1 \mu \mathrm{g} / \mathrm{ml}$, respectively. All the patients who were given lidocaine showed disturbances of the central nervous system such as dysarthria and light headedness.

The induction of sustained VT was suppressed in 15 of 29 patients (52\%) by procainamide, but in none by lidocaine (Table II). The induction of nonsustained VT was suppressed in 6 of 18 patients (33\%) by procainamide, and in 1 of 8 patients $(13 \%)$ by lidocaine (Table II). The efficacy of procainamide in suppression of VT induction was significantly higher than that of lidocaine $(p<0.01)$ (Table II). Fig. 1 shows the results of programmed electrical stimulation of a patient in whom both lidocaine and procainamide were tested respectively, and the former

$$
\text { Japanese Circulation Journal Vol. 52, March } 1988
$$




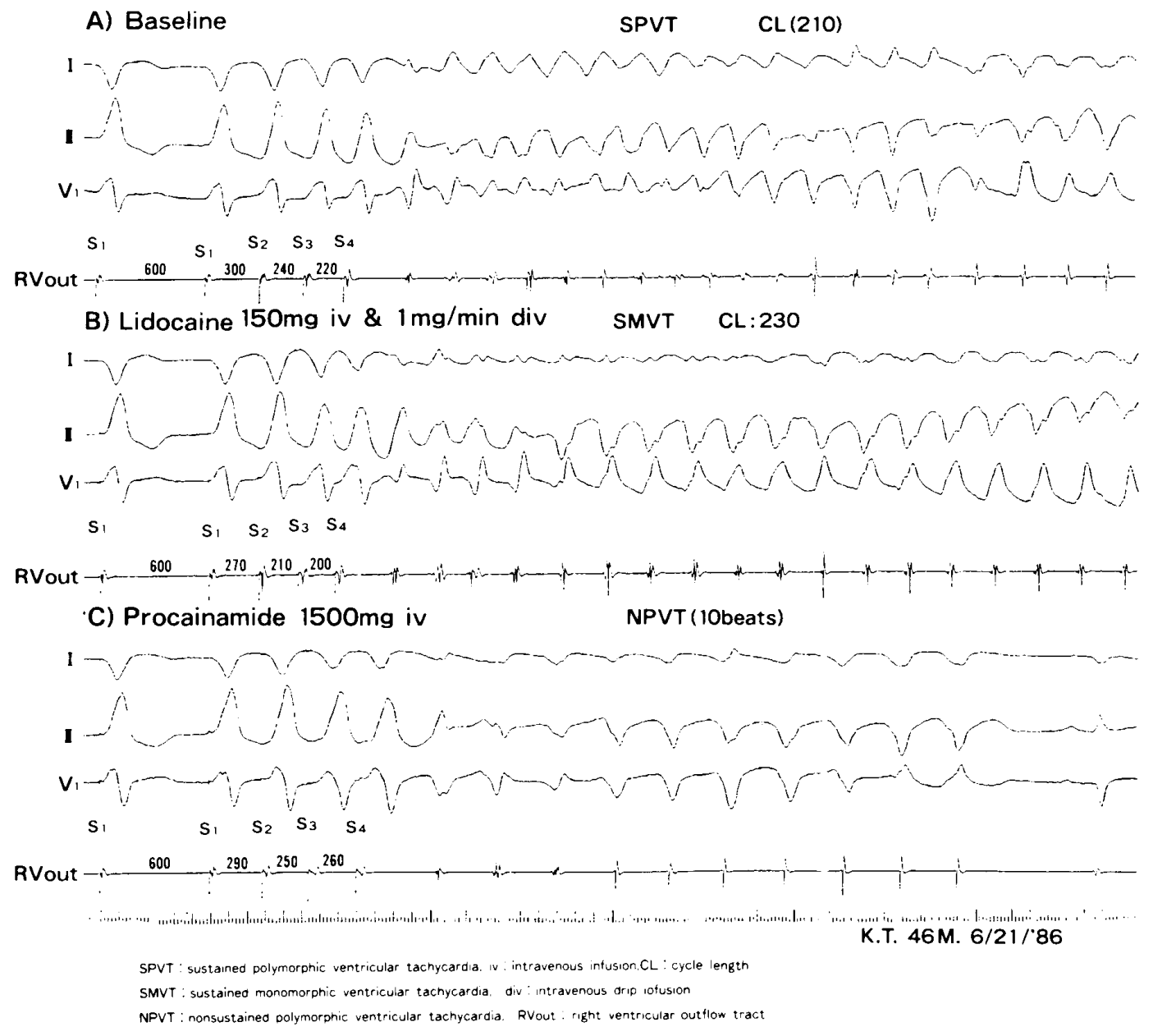

Fig.1. Demonstration of a case in whom procainamide was shown to be effective while lidocaine to be ineffective in suppression of sustained VT induction by serial drug testing using programmed ventricular stimulation. Three surface electrocardiograms and one intracardiac electrogram from the right ventricular outflow tract (RV out) are recorded simultaneously. (A) In baseline, sustained polymorphic VT (SPVT) was induced by triple extrastimuli (S1, S2, S3, S4). (B) Post lidocaine sustained monomorphic VT (SMVT) remained inducible by triple extrastimuli. (C) Procainamide suppressed the induction of sustained VT and nonsustained VT of more than 10 beats.

proved to be ineffective, but the latter to be effective.

The electrophysiological effects of lidocaine and procainamide were compared (Fig. 2). The mean effective refractory periods of the right ventricle measured by the first extrastimulus (ERP-S2), the second extrastimulus (ERP-S3), and the third extrastimulus (ERP-S4) were significantly prolonged by procainamide compared to the baseline state (ERP-S2: $244 \pm 23$ $\mathrm{ms}$ vs $272 \pm 26 \mathrm{~ms}$, ERP-S $3: 196 \pm 28 \mathrm{mg}$ vs $230 \pm 34 \mathrm{~ms}$, ERP-S4: $186 \pm 25 \mathrm{~ms}$ vs $218 \pm 30$ $\mathrm{ms}, \mathrm{p}<0.01)$. The mean cycle length of induced VT was also prolonged significantly by procainamide compared to that before the drug application $(240 \pm 49 \mathrm{~ms}$ vs $304 \pm 61 \mathrm{~ms}, \mathrm{p}<0.01)$.
In addition the mean HV interval, QRS interval during sinus rhythm and ventricular pacing at the same cycle length, and QTc interval were significantly prolonged by procainamide (HV interval: $46 \pm 7 \mathrm{~ms}$ vs $56 \pm 12 \mathrm{~ms}$, QRS interval during sinus rhythm: $98 \pm 20 \mathrm{~ms}$ vs $109 \pm 23 \mathrm{~ms}$, QRS interval during ventricular pacing: $172 \pm 12$ $\mathrm{ms}$, vs $194 \pm 17 \mathrm{~ms}$, QTc interval: $412 \pm 31 \mathrm{~ms}$ vs $478 \pm 34 \mathrm{~ms}, \mathrm{p}<0.01)$. On the other hand lidocaine did not affect these electrophysiological parameters significantly.

In a certain number of cases, either one or both drugs showed worsening effects on the induction of arrhythmias. Fig. 3 shows a case in which the worsening effect of both lidocaine and procainamide were demonstrated. In this case 


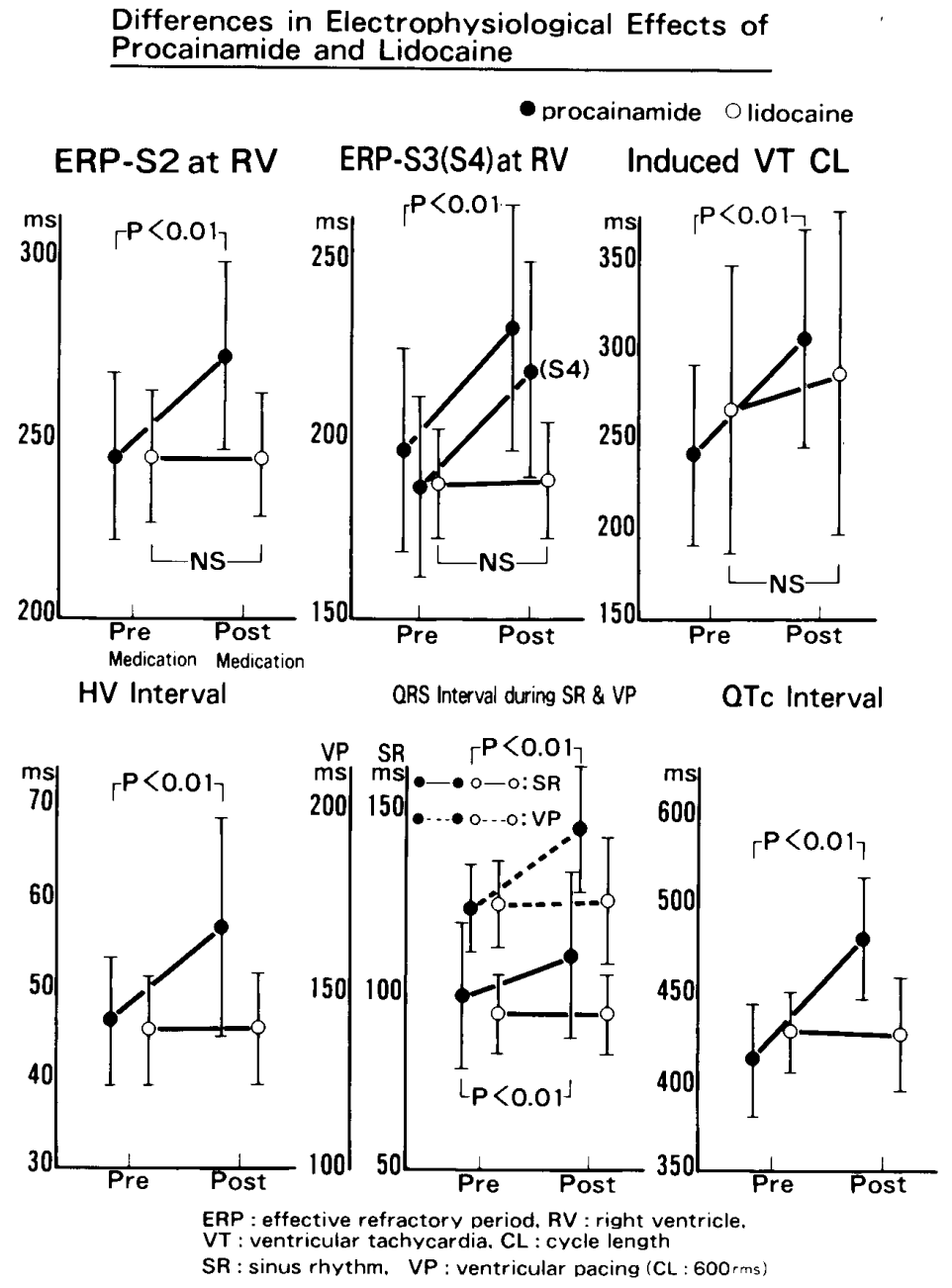

Fig.2. Comparisons in electrophysiological effects between procainamide and lidocaine. The mean effective refractory periods of the right ventricle measured by the first extrastimulus (ERP-S2 at RV), the second extrastimulus (ERP-S3 at RV), and the third extrastimulus (ERP-S4 at RV), the induced VT cycle length (CL), the HV interval, the QRS interval during sinus rhythm (SR) and ventricular pacing (VP), and the QTc interval were all significantly prolonged by procainamide $(\bullet)$, while they were not changed significantly by lidocaine $(0)$.

nonsustained polymorphic VT was changed into sustained monomorphic VT to become inducible. The worsening effect was demonstrated in 8 of 18 cases (44\%) with procainamide administration, and in 3 of 8 cases $(38 \%)$ with lidocaine. There were no differences in the incidence of worsening effect between the two drug groups. Morphologic changes of induced VT from polymorphic in to monomorphic form were seen in 5 of 6 cases (83\%) of procainamide, and in 3 of 5 cases $(60 \%)$ of lidocaine. In all of these cases, both drugs were proven to be ineffective or to aggravate arrhy thmias.

Electrophysiological effects of procainamide were compared between the procainamide effective ( 21 cases, group I) and the ineffective or worsening (26 cases, groups II) patients (Fig. 4). There were no significant differences between the two groups either in the baseline electrophysiologic parameters or those during the drug application. However, there was a tendency for the degrees of prolongation in the effective refractory periods of the right ventricle and QRS interval to be larger in group I than group II ( $\triangle$ ERP-S2: $30 \pm 16 \mathrm{~ms}$ vs $23 \pm 17 \mathrm{~ms}$, $\triangle$ ERP-S3: $38 \pm 24 \mathrm{~ms}$ vs $30 \pm 24 \mathrm{~ms}, \triangle$ ERP-S4: $40 \pm 18 \mathrm{~ms}$ vs $18 \pm 21 \mathrm{~ms}, \Delta \mathrm{QRS}: 16 \pm 19 \mathrm{~ms}$ vs $8 \pm 8 \mathrm{~ms}, \mathrm{P}=\mathrm{NS}$ ).

In 3 cases the combined effects of procainamide and lidocaine were studied. In 1 case, induction of sustained VT, which was inducible with 3 extrastimuli in the baseline, was abolished 


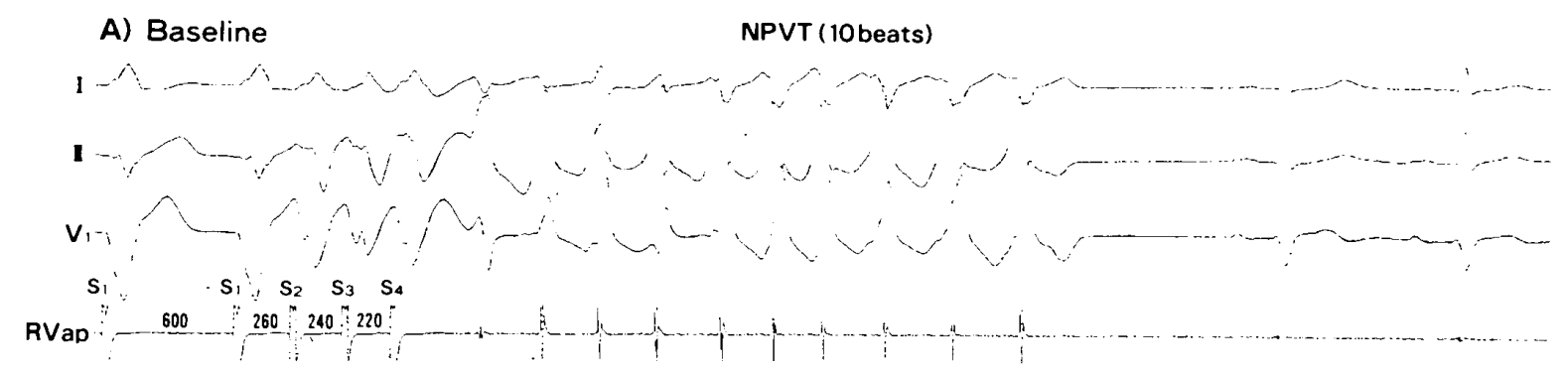

B) Lidocaine $180 \mathrm{mg}$ iv $\& 2 \mathrm{mg} / \mathrm{min}$ div SMVT

$C L: 230$

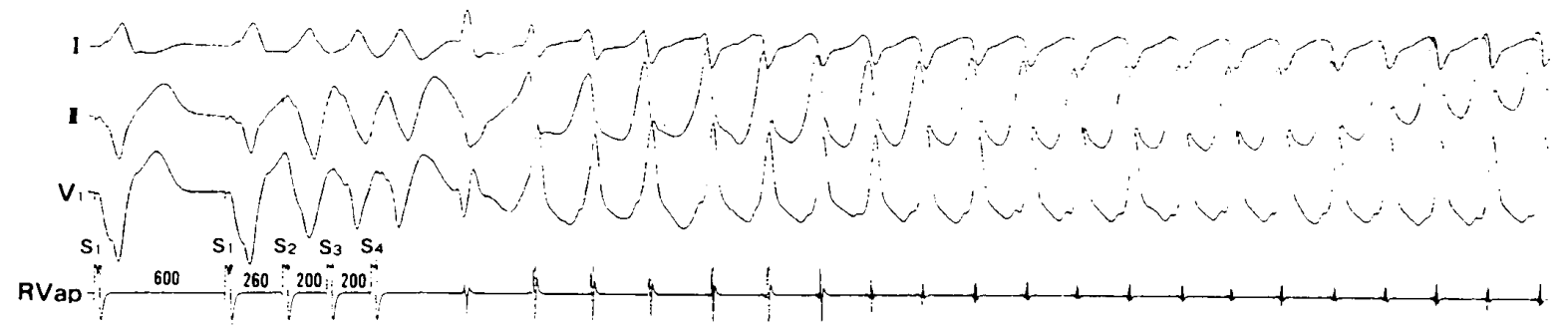

C) Procainamide $1000 \mathrm{mg}$ iv

SMVT

$\mathrm{CL}: 230$

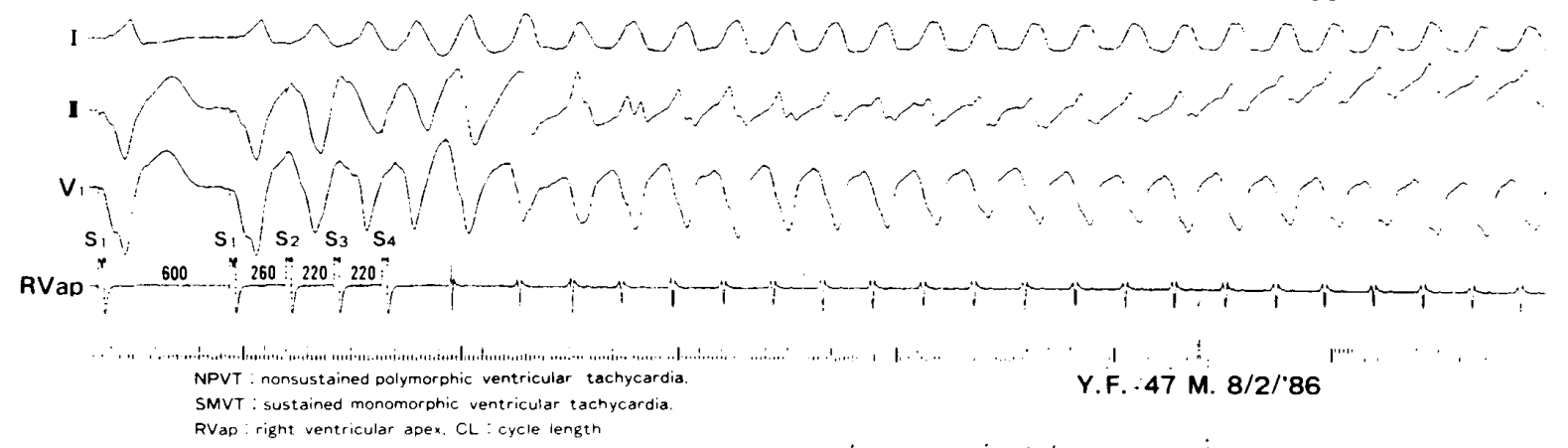

Fig.3. Demonstration of a case in whom the worsening effects of procainamide and lidocaine were shown by serial drug testing. (A) In baseline, nonsustained polymorphic VT (NPVT) of 10 beats was inducible by triple extrastimuli. (B) (C) Post lidocaine and then procainamide, sustained monomorphic VT (SMVT) became to be inducible.

by procainamide, while sustained VT became inducible with only 2 extrastimuli after lidocaine was added. In the remaining 2 cases, the lidocaine application combined with procainamide did not enhance the suppressive effect of procainamide on VT induction, but it cancelled a prolonging effect of the effective refractory period of the right ventricle produced by procainamide to some degree.

\section{DISCUSSION}

Antiarrhythmic drug therapy still stands as the main therapeutic intervention for prevention of recurrent life-threatening ventricular tachyarrhythmias: At the present time, there are two major approaches to evaluating antiarrhythmic drug effects; the noninvasive and the invasive methods. A noninvasive approach, using repeated Holter monitoring and/or exercise testing is based on the detection of spontaneous ventricular ectopic: activity with complexity and high frequency. The rationale for employing this approach is that the abolition of triggering ventricular arrhythmias will prevent the occurrence of life-threatening ventricular tachyarrhythmias. The absence of frequent ventricular arrhythmias precludes the use of this noninvasive method. It is well known that a certain number of cases with recurrent sustained ventricular tachycardia do not have frequent ventricular arrhy thmias detectable by Holter monitoring.4,25 In addition, the abolition of triggering arrhythmias as a goal of this method has not yet been established as a fully effective way to prevent recurrent sustained ventricular tachycardias. ${ }^{26}$

The invasive approach is an electrophysiological test using programmed ventricular stimulation. The method has been used to identify the potential high-risk patients for sudden cardiac 
Comparison of Electrophysiological Effects of Procainamide between Effective and Ineffective/Worsening Groups

- procainamide effective. M procainamide ineffective or worsening

ERP-S2

ERP-S3

ERP-S4
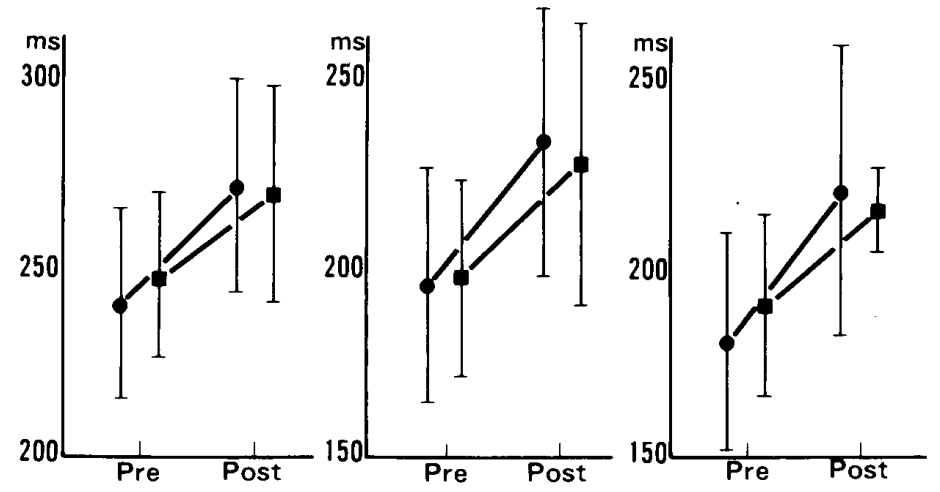

HV Interval

QRS Interval

QTc Interval
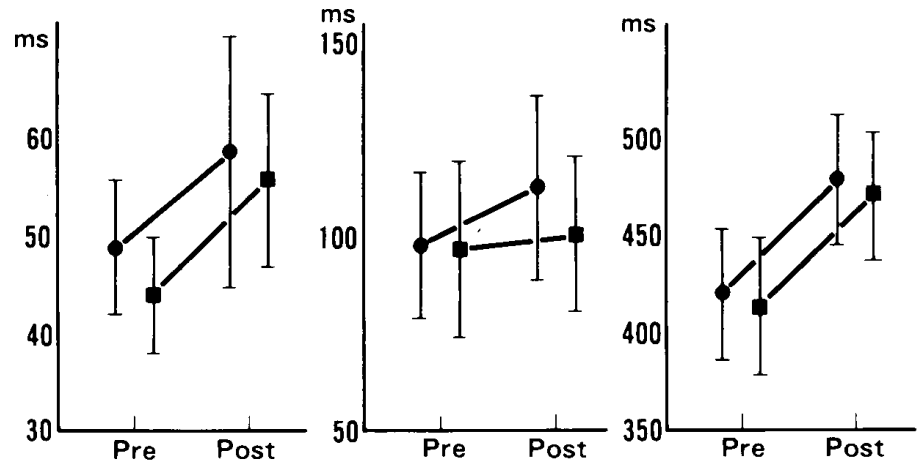

Fig.4. Comparison of electrophysiological effects of procainamide between the procainamide effective and ineffective or worsening groups. There was a tendency that the degrees of prolongation in the effective refractory periods of the right ventricle (ERP-S2, ERP-S3, ERP-S4) and QRS interval were larger in the procainamide effective group $(\bullet)$ than the ineffective or worsening group ( $\bullet$ ).

death including those who have had myocardial infarction. The method has also been used to select and prospectively evaluate antiarrhythmic regimens for treatment of recurrent sustained VT. ${ }^{1-24}$ In several studies including ours, the inducibility of sustained ventricular tachycardia has been shown to be useful in predicting the occurrence of spontaneous sustained ventricular tachycardia or sudden cardiac death, $90,18,27$ although the predictive value and clinical significance of this technique remaind to be determined. There is no way other than programmed electrical stimulation to disclose the substrate which allows the development of unidirectional block and delayed conduction essential to initiate and perpetuate reentrant tachyarrhythmias. The method is also useful in examining the effect of drugs on the reentry circuit. We investigated the effects of the most widely used antiarrhythmic agents, procainamide and lidocaine, on electrically inducible VT. The study was con- ducted in patients at the post-acute phase of myocardial infarction in an effort to evaluate the usefulness and limitations of medical treatment in preventing life-threatening ventricular tachyarrhythmias.

In this study, the efficacy rate of procainamide was $45 \%$ in total VTs ( $52 \%$ in sustained VT and $33 \%$ in nonsustained VT), while that of lidocaine was $7 \%$ (0\% and $13 \%$, respectively). Procainamide was significantly more effective than lidocaine. These results are similar to those of other reports ${ }^{24,28-37}$ in which the efficacy rate of acute intravenous procainamide administration ranges from 13 to $48 \%, 24,28-37$ and that of lidocaine from 5 to $19 \%, 24,28,31$ The variability in the results seems to be due to differences in patient selection, the stimulation protocol, the doses of the applied drugs and the evaluation criteria for drug efficacy. In our study, the patients were at the post-acute phase of myocardial infarction and most had no documented 
episodes of recurrent sustained VT. The induced VT included both sustained and nonsustained type, and triple extrastimuli were used to examine inducibility of VT. In addition, VT was defined as 10 or more consecutive ventricular beats, and the drug was judged as effective even when sustained VT was changed into nonsustained VT inducible.

The mechanism of the majority of VT in the present study appears to be reentry, since they were repeatedly induced by the extrastimuli with certain limits of the coupling intervals. Some of them were also terminated by the extrastimuli (Table I). It is not completely ruled out, however, that abnormal impulse formation such as triggered-activity as a cause of the VT may play a role in the genesis of the induced arrhythmias in some of the cases. Procainamide is presumed to prevent reentrant tachyarrhythmias either by slowing conduction velocity in the area of depressed conduction and converting unidirectional block into bidirectional one, by prolonging refractory period in the reentry pathway or by a combination of both $29,33,34,36-38$ In this study, while the effective refratory period of the right ventricle as well as the HV and QRS intervals reflecting intraventricular conduction time were significantly prolonged by procainamide, no correlation was found between the effects on VT inducibility and those on the effective refractory periods and the indicators of intraventricular conduction. These results agree with those reported by others. ${ }^{34,36,37}$ This may be explained by the fact that prolongation of the refractory period and conduction velocity were measured in the global ventricular tissue, and may not accurately reflect the effect within the reentry circuit of $\mathrm{VT}^{34,36,37}$ At present it is not possible to evaluate the effects of antiarrhythmic agents on the reentry circuit itself. On the other hand lidocaine did not show any prolonging effects on either the refractory periods or the conduction velocity, which may explain different effects on the prevention of the VT inducibility between the two drugs.

In a considerable number of cases, nonsustained VT were converted into sustained VT inducible after the drug application. These proarrhythmic effects were demonstrated in $44 \%$ cases of the procainamide group and $38 \%$ of the lidocaine group. The incidence of proarrhythmic effects by procainamide has been reported to be in a range between 13 and
$20 \% 29,34,35,38,39$ The differences between our data and those of others are mostly attributed to the patient selection and the difference in the pacing protocol. The proarrhythmic effects of lidocaine have not been described in the literature and remaind to be investigated. The potential mechanism of the proarrhythmic actions by the class I agents is indicated by their effects on the conduction velocity and the refractory period, both of which are related to their antiarrhythmic actions. Therefore, the proarrhythmic effects of these agents cannot be ignored whenever they are used.

In summary, the study demonstrated that procainamide representing class IA drugs was more effective than lidocaine representing class IB drug for the prevention of a potential lifethreatening ventricular tachycardia in patients post myocardial infarction. However, its efficacy appeared to be considerably limited. Drug testing using programmed ventricular stimulation was shown to be a valuable method to confirm the effectiveness of antiarrhythmic agents and to exclude their proarrhythmic actions in each patients.

\section{REFERENCES}

1. MOSS AJ, DECAMILla J, DAVIS HT: Cardiac death in the first 6 months after myocardial infarction: Potential for mortality reduction in the early post-hospital period. Am J Cardiol 39: 816, 1977

2. KANNEL W, STOLliE P, McNAMARA P: Prognosis after initial myocardial infarction: The Framingham study. Am J Cardiol 44: 53, 1979

3. HOROWITZ LN, MORGANROTH J: Can we prevent sudden cardiac death? Am J Cardiol 50: 535,1982

4. MARCHLINSKI FE, BUXTON AE, WAXMAN HL, JOSEPHSON ME: Role of electrophysiologic study in predicting sudden death following myocardial infarction. In Tachycardias, ed by JOSEPHSON ME and WELLENS HJJ, Lea \& Febiger, Philadelphia, 1984, p 497

5. MOSS AJ, DAVIS HT, DECAMILLA J, BAYER LW: Ventricular ectopic beats and their relation to sudden and nonsudden cardiac death after myocardial infarction. Circulation 60: 998, 1979

6. SCHULZE RA, STRAUSS HW, PITT B: Sudden death in the year following myocardial infarction: Relation to ventricular premature contractions in the late hospital phase and left ventricular ejection fraction. Am JMed 62: 192, 1977

7. BIGGER JT, COROMILAS J, WELD FM, REIFFEL JA, ROLNITZKY LM: Identification of high-risk patients after myocardial infarction: Pointers for management. In Sudduen Cardiac Death, ed by MORGANORTH J, and HOROWITZ LN, Grune \& Stratton, Orlando, 1985, p 109

8. GREEN HL, REID PR, SCHAEFFER AH: The

Japanese Circulation Journal Vol. 52, March ${ }_{1988}$ 
repetitive ventricular response in man: A predictor of sudden death. N Engl J Med 299: 729, 1978

9. HAMER A, VOHRA J, HUNT D, SLOMAN JG: Prediction of sudden death by electrophysiologic studies in high risk patients surviving acute myocardial infarction. Am J Cardiol 50: 223, 1982

10. RICHARDS DA, CODY DV, DENNIS AR, RUSSELL PA, YOUNG AA, UTHER JB: Ventricular electrical instability: a predictor of death after myocardial infarction. Am J Cardiol 51: 75, 1983

11. MARCHLINSKI FE, BUXTON AE, WAXMAN HL, JOSEPHSON ME: Identifying patients at risk of sudden death after myocardial infarction: value of the response to programmed stimulation, degree of ventricular ectopic activity, and severity of left ventricular dysfunction. Am J Cardiol 52: 1190 , 1983

12. BREITHARDT G, BORGGREFE M, HAERTEN $\mathrm{K}$ : Role of programmed ventricular stimulation and non-invasive recording of ventricular late potentials for the identification of patients at risk of ventricular tachyarrhythmias after acute myocardial infarction. In Cardiac Electrophysiology and Arrhy thmias, ed by ZIPES $D$ and JOLIFE J, Grune \& Stratton, New York, 1983, p 553

13. SANTARELLI P, BELLOCCI F, LOPERFIDO F, MAZZARI M, MONGIARDO R, MONTENERO A, MANZOLI V, DENES P: Ventricular arrhythmia induced by programmed ventricular stimulation after acute myocardial infarction. Am $J$ Cardiol 55: 391,1985

14. WASPE LE, SEINFIELD D, FERRICK A, KIM SG, MATOS JA, FISHER JD: Prediction of sudden death and spontaneous ventricular tachycardia in survivors of complicated myocardial infarction: value of the response to programmed stimulation using a maximum of three ventricular stimuli. $J$ Am Coll Cardiol 5: 1292, 1985

15. ROY D, MARCHAND E, THEROUX P, WATERS DD, PELLETIER GB, BOURASSA MG: Programmed ventricular stimulation in survivors of an acute myocardial infarction. Circulation 72: 487, 1985

16. SANTARELLI P, BELLOCCI F, LOPESFIDO F, SANDRIC S, DENES P: Identification of patients at risk of sudden death after myocardial infarction: Is there a role for programmed ventricular stimulation? Clin Prog Electorophysiol Pacing 3: 115, 1985

17. FRIEDMAN L, YASUF S: Does therapy directed by programmed electrical stimulation provide a satisfactory clinical response? Circulation 73 (Suppl II): 59, 1986

18. DENNISS AR, RICHARDS DA, CODY DV, RUSSELL PA, YOUNG AA, COOPER MJ, ROSS DL, UTHER JB: Prognostic significance of ventricular tachycardia and fibrillation induced at programmed stimulation and delayed potentials dected on the signal averaged electrograms of survivors of acute myocardial infarction. Circulation 74: 731, 1986

19. UTHER JB, RICHARDS AB, DENNIS AR, ROSS DL: The prognostic significance of programmed ventricular stimulation after myocardial infarction: A review. Circulation 75 (Suppl III): 161, 1987

20. BRUGADA P, WALDECKER B, KERSSCHOT Y,
ZEHENDER M, WELLENS HJJ: Ventricular arrhythmias initiated by programmed stimulation in four groups of patients with healed myocardial infarction. J Am Coll Cardiol 8: 1035, 1986

21. FISHER JD, COHEN HL, MEHRA R, ALTSCHULER H, ESCHER DJW, FURMAN S: Cardiac pacing and pacemakers. II. Serial electrophysiologic-pharmacologic testing for control of recurrent tachyarrhy thmias. Am Heart J 93: 658, 1977

22. MASON JM, WINKLE RA: Electrode-Catheter arrhythmia induction in the selection and assessment of antiarrhythmic drug therapy for recurrent ventricular tachycardia. Circulation 58: 971, 1978

23. HOROWITS LN, JOSEPHSON ME, FARSHIDI A, SPIELMAN SR, MICHELSON EL, GREENSPAN AM: Recurrent sustained ventricular tachycardia. 3. Role of the electrophysiologic study in selection of antiarrhythmic regimens. Circulation 58: 986, 1978

24. WAXMAN HL, BUXTON AE, MARCHLINSKI FE, JOSEPHSON ME: Pharmacologic therapy of sustained ventricular tachyarrhythmias. In Tachycardias, ed by JOSEPHSON ME and WELLENS HJJ, Lea \& Febiger, Philadelphia, 1984, p 497

25. BIGGER JT, REIFFEL JA: Holter versus electrophysiologic studies in the management of malignant ventricular arrhythmias. Am J Cardiol 51: 1464,1983

26. MORGANROTH J: Ambulatory electrocardiographic monitoring into evaluation of near antiarrhythmic drugs. Circulation 73 (Suppl II): 92, 1986

27. IESAKA Y, AONUMA K, NITTA J, TOKUNAGA T, ZEN E, SHIROYAMA N, FUJIWARA $\mathrm{H}$ : Predictive importance of sustained monomorphic ventricular tachycardia induced in predischarge myocardial infarction. $P A C E$ 10: 407, 1987 (abst)

28. HOROWITZ LN, JOSEPHSON ME, KASTOR JA: Intracardiac electrophysiologic studies as a method for the optimization of drug therapy in chronic ventricular arrhythmia. Prog Cardiovasc Dis 23: 81,1980

29. GREENSPAN AM, HOROWITZ LN, SPIELMAN SR, JOSEPHSON ME: Large dose procainamide therapy for ventricular tachyarrhythmia. $A m J$ Cardiol 46: 453, 1980

30. SWERDLOW CD, ECHT DS, WINKLE RA, GRIFFIN JC, ROSS DL, MASON JW: Incidence of acute antiarrhythmic drug efficacy at electropysiologic study. Circulation 64 (Suppl IV): 137, 1981 (abst)

31. ROSS DL, SZE DY, KEEFE DL, SWERDLOW CD, ECHT DS, GRIFFIN JC, WINKLE RA, MASON JW: Antiarrhythmic drug combinations in the treatment of ventricular tachycardia: Efficacy and electrophysiologic effects. Circulation 66: 1205, 1982

32. SWERDLOW CD, BLUM J, WINKLE RA, GRIFFIN JC, ROSS DL, MASON JW: Decreased incidence of antiarrhythmic drug efficacy at electrophysiologic study associated with the use of a third extrastimulus. Am Heart J 104: 1004, 1982

33. WAXMAN HL, BUXTON AE, SADOWSKI LM, JOSEPHSON ME: The response to procainamide during electrophysiologic study for sustained ventricular tachyarrhythmias predicts the response 
to other medications. Circulation 67: 28, 1983

34. MARCHLINSKI FE, BUXTON AE, VASSALLO JA, WAXMAN HL, CASSIDY DM, DOHERTY JU, JOSEPHSON ME: Comparative electrophysiologic effects of intravenous and oral procainamide in patients with sustained ventricular arrhythmias. J Am Coll Cardiol 4: 1247, 1984

35. RAE AP, GREENSPAN AM, SPIELMAN SR, SOKOLOFF NM, WEBB CR, KAY HR, HOROWITZ LN: Antiarrhythmic drug efficacy for ventricular tachyarrhythmias associated with coronary artery disease as assessed by electrophysiologic studies. Am J Cardiol 55: 1494, 1985

36. MORADY F, DiCARLO LA, BUITLEIR M, KROL RB, BAERMAN JM, KOU WH: Effects of incremental doses of procainamide on ventricular refractoriness, intraventricular conduction, and induction of ventricular tachycardia. Circulation 74: 1355,1986

37. GOLD RL, HAFFAJEE CI, ALPERT JS: Electrophysiologic and clinical factors influencing response to class IA antiarrhythmic agents in patients with inducible sustained monomorphic ventricular tachycardia. Am Heart J 112: 9, 1986

38. AU PK, BHANDARI AK, BREAM R, SCHRECK D, SIDDIQI R, RAHIMTOOLA SH: Proarrhy thmic effects of antiarrhythmic drugs during programmed ventricular stimulation in patients without ventricular tachycardia. J Am Coll Cardiol 9: 389, 1987

39. VELEBIT V, PODRID P, LOWN B, COHEN BH, GRABOYS TB: Aggravation and provocation of ventriculat arrhythmias by antiarrhythmic drugs. Circulation 65: 886, 1982 